\title{
TOPOLOGICAL PROPERTIES OF THE SCALE OF A UNIFORM SPACE
}

\author{
G. D. RICHARDSON and E. M. WOLF
}

(Received 2 September 1980)

Communicated by J. H. Rubinstein

\begin{abstract}
Let $(S, Q L)$ be a uniform space. This space can be embedded in a complete, uniform lattice called the scale of $(S, \mathscr{Q})$. We prove that the scale is compact if and only if $S$ is finite or $\mathscr{Q}=\{S \times S\}$. We prove that this statement remains true if compact is replaced by countably compact, totally bounded, Lindelof, second countable, or separable. In the last section of this paper, we investigate the cardinality of the scale and the retracted scale.
\end{abstract}

1980 Mathematics subject classification (Amer. Math. Soc.): 54 D 30, 54 D 45, 54 E 15.

Keywords and phrases: uniform space, scale, retracted scale, compact, totally bounded, separable, cardinality.

\section{Introduction}

Kent [3] showed that each uniform space $(S, \mathcal{Q})$ could be embedded in a complete, uniform lattice called the scale of $(S, \mathscr{U})$. The scale was first introduced by Bushaw [2] for studying stability in topological dynamics. The local connectedness and connectedness of the scale were studied in [4]. In [5] it was shown how these results apply to uniform subspaces of $\mathbf{R}$. In this paper we consider cases where the scale is compact. We prove that " $S$ is finite or $थ=\{S \times S\}$ " is equivalent to the scale being countably compact, totally bounded, Lindelöf, compact, second countable, and separable.

We also consider the retracted scale which is obtained from the scale by a quotient construction. The retracted scale is a complete uniform lattice which is

C) Copyright Australian Mathematical Society 1982 
always Hausdorff. We prove that if $S$ is infinite and $\mathcal{Q} \neq\{S \times S\}$, then the scale and the retracted scale both have cardinality $2^{\text {(ard }(S)}$.

Given any nonempty set $S$, a prefilter on $S$ is a nonempty collection $\alpha$ of subsets of $S$ which is closed with respect to supersets. Suppose $(S, \mathscr{Q})$ is a uniform space. Let $P$ be the set of all prefilters on $S \times S$ which are subsets of $\mathcal{Q}$. For any two elements $\alpha$ and $\beta$ in $P$, define $\alpha \leqslant \beta$ to mean $\alpha \supset \beta$. The structure $(P, \leqslant)$ is called the scale of $(S, \mathcal{Q}) .(P, \leqslant)$ is a complete distributive lattice whose suprema and infima are set intersections and set unions, respectively.

If $U$ and $V$ are two subsets of $S \times S$, then the set of all pairs $(x, y) \in S \times S$ such that $(x, z) \in V$ and $(z, y) \in U$ for some $z \in S$ is denoted by $U \circ V$. For each $U \in \mathscr{Q}$, let $U^{\prime}=\{(\alpha, \beta) \in P \times P \mid A \in \alpha$ implies $U \circ A \in \beta$, and $B \in \beta$ implies $U \circ B \in \alpha$ \}. The following proposition is proved in [3, page 160].

Proposition 1. For each $U \in \mathcal{Q}, U^{\prime 2}=U^{2^{\prime}}$.

Each set of the form $U^{\prime}$ is symmetric and contains the diagonal of $P \times P$; thus $\left\{U^{\prime} \mid U \in \mathscr{Q}\right\}$ is the base for a uniformity on $P$, denoted by $\mathcal{T}$. We call $(P, \mathcal{T})$ the scale of the uniform space associated with $(S, \mathcal{Q})$.

Next we construct the retracted scale. Let $R$ be an equivalence relation on $P$ defined by: $(\alpha, \beta) \in R$ whenever $(\alpha, \beta) \in U^{\prime}$, all $U \in \mathcal{Q}$. Let $\alpha_{R}$ denote the $R$ equivalence class containing $\alpha$. In [3, page 160] it is shown that

$$
\alpha_{R}=\left\{\beta\left|\alpha_{0} \leqslant \beta\right| \alpha^{0}\right\}
$$

where

$$
\begin{aligned}
& \alpha_{0}=\{U \in \mathcal{Q} \mid V \circ U \in \alpha, \text { all } V \in \mathcal{Q}\}, \text { and } \\
& \alpha^{0}=\{U \in \mathcal{Q} \mid v \circ A \subset U \text { for some } V \in \mathcal{Q} \text { and some } A \in \alpha\} .
\end{aligned}
$$

Let $P_{R}=\left\{\alpha_{R} \mid \alpha \in P\right\}$. Given $\alpha, \beta$ in $P_{R}$ we define $\alpha \leqslant \beta$ to mean $\alpha_{0} \supset \beta_{0}$. The order structure $\left(P_{R}, \leqslant\right)$ is called the retracted scale of $(S, \mathcal{Q})$. Let the canonical mapping from $P$ to $P_{R}$ be denoted by $\psi$ so that for $\alpha \in P, \psi(\alpha)=\alpha_{R}$.

To define the retracted scale uniformity, we recall that with each $U \in \mathcal{Q}$ there is an associated entourage $U^{\prime}$ in the scale uniformity $\mathscr{T}$. Let $U^{\prime \prime}=\psi\left(U^{\prime}\right)=$ $\left\{\left(\alpha_{R}, \beta_{R}\right) \mid(\alpha, \beta) \in U\right\}$. The filter $\mathfrak{T}_{R}$ generated by $\left\{U^{\prime \prime} \mid U \in \mathscr{Q}\right\}$ is a uniformity. We call $\left(P_{R}, \mathcal{V}_{R}\right)$ the retracted scale uniform space associated with $(S, \mathcal{U})$. 
We will find it convenient to use the following closed interval notation. For $\alpha \leqslant \beta$, let $[\alpha, \beta]=\{\gamma \in P \mid \alpha \leqslant \gamma \leqslant \beta\}$. For each $U \in \mathcal{Q}$ and $\alpha \in P$ let

$$
\begin{aligned}
& \alpha_{U}=\{V \in \mathcal{Q} \mid U \circ V \in \alpha\} \text { and } \\
& \alpha^{U}=\{V \in \mathcal{Q} \mid \text { for some } A \in \alpha, U \circ A \subset V\} .
\end{aligned}
$$

It is easily shown that $U^{\prime}(\alpha)=\left[\alpha_{U}, \alpha^{U}\right]$. This implies the next proposition.

Proposition 2. The collection $\left\{\left[\alpha_{U}, \alpha^{U}\right] \mid U \in \mathcal{Q}\right\}$ forms a basic system of neighborhoods at $\alpha$ for the uniform topology on $P$ generated by $\mathcal{\Upsilon}$.

Suppose that $\alpha=\mathcal{Q}$. Then $\alpha$ is the least element of $P$, and we denote it by 0 . Observe that $0_{U}=0$. Let $\langle U\rangle$ denote the prefilter consisting of all oversets of $U$. Since $0^{V} \leqslant\langle U\rangle \leqslant 0^{U}$ for $V^{2} \subset U$, Proposition 2 implies that the collection $\{[0,\langle U\rangle] \mid U \in \mathscr{Q}\}$ is a basic neighborhood system of 0 .

The next proposition will be useful in proving that a necessary and sufficient condition for the scale to have a totally bounded neigborhood of 0 is that $\mathcal{U}$ have a least element.

PROPOSITION 3. Suppose that ( $S$, थ) is a uniform space such that there exists a $U \in \mathcal{Q}$ with the property that the complement of $U$ is an infinite set. Then either $\mathcal{Q}$ has a least element or there exists a countably infinite discrete subspace of $[0,\langle U\rangle]$.

Proof. Assume that $\mathcal{Q}$ does not have a least element. Then there exists $V=V^{-1} \in \mathcal{Q}$ and $W=W^{-1} \in \mathcal{Q}$ such that $W^{3} \subset V \subset V^{2} \subset U$ and $W^{3} \neq V$. Let us assume without loss of generality that $U=U^{-1}$ and $\left(a_{n}, b_{n}\right) \in S \times S-U$, where $a_{n} \neq a_{m}$ whenever $n \neq m$. For $n \geqslant 1$, define $\beta_{n}=\langle U\rangle \cup\left\langle W \cup\left\{\left(a_{n}, b_{n}\right)\right\}\right\rangle$. Then $A=\left\{\beta_{n} \mid n \geqslant 1\right\}$ is a subset of $[0,\langle U\rangle]$. Let $\alpha \in P$; then $W^{\prime}(\alpha)$ is a neighborhood of $\alpha$ in $P$. Suppose that $\beta_{k}, \beta_{l} \in W^{\prime}(\alpha)$; then $\left(\beta_{k}, \beta_{l}\right) \in\left(W^{\prime}\right)^{2}=$ $\left(W^{2}\right)^{\prime}$ by Proposition 1. Thus either

$$
\begin{gathered}
W^{2} \circ\left(W \cup\left\{\left(a_{k}, b_{k}\right)\right\}\right) \supset U, \text { or } \\
W^{2} \circ\left(W \cup\left\{\left(a_{k}, b_{k}\right)\right\}\right) \supset W \cup\left\{\left(a_{l}, b_{l}\right)\right\} .
\end{gathered}
$$

Suppose that (i) holds and let $(a, b) \in V-W^{3}$. Then $(a, b) \in W^{2} \circ[W \cup$ $\left.\left\{\left(a_{k}, b_{k}\right)\right\}\right]$. There exists a $c \in S$ such that $(a, c) \in W \cup\left\{\left(a_{k}, b_{k}\right)\right\}$ and $(c, b) \in$ $W^{2}$. Since $(a, b) \notin W^{3},(a, c)=\left(a_{k}, b_{k}\right)$ or $a=a_{k}, c=b_{k}$. Hence $\left(b, b_{k}\right) \in W^{2}$ and $\left(a_{k}, b\right) \in V$ so $\left(a_{k}, b_{k}\right) \in V^{2} \subset U$, contrary to the choice of the $\left(a_{n}, b_{n}\right)$ 's. 
Suppose (ii) holds. Then $\left(a_{l}, b_{l}\right) \in W^{2} \circ\left[W \cup\left\{\left(a_{k}, b_{k}\right)\right\}\right]$ implies that there exists a $c \in S$ such that $\left(a_{l}, c\right) \in W \cup\left\{\left(a_{k}, b_{k}\right)\right\}$ and $\left(c, b_{l}\right) \in W^{2}$. Since $\left(a_{l}, b_{l}\right) \notin W^{3}, a_{l}=a_{k}$ and thus $l=k$, and $\beta_{k}=\beta_{l}$. It follows that $W^{\prime}(\alpha)$ contains at most one point of $A$, and so $A$ is a discrete subset of $P$.

Proposition 4. Let $(S, \mathscr{Q})$ be a uniform space. Then $(P, \mathcal{T})$ has a totally bounded neighborhood of 0 if and only if थ has a least element.

Proof. If $U \in \mathcal{U}$ is a least element in $\mathcal{Q}$, then the set $[0,\langle U\rangle]=\{0\}$ is a totally bounded neighborhood of 0 . Conversely, suppose that $(P, \mathscr{V})$ has a totally bounded neighborhood of 0 . It then follows from remarks preceding Proposition 3 that there exists a $U \in \mathscr{Q}$ such that $[0,\langle U\rangle]$ is totally bounded. If $S$ is a finite set, $\mathcal{Q}$ has a least element. Suppose that $S$ is an infinite set. Let $V \in \mathcal{Q}$ be any subset of $Q$. Since $[0,\langle V\rangle]$ is totally bounded, it follows from Proposition 3 , that either $\mathcal{Q}$ has a least element or $S \times S-V$ is a finite set. Assume that $V=V^{-1}$ and $S \times S-V$ is a finite set; then for any $a, b \in S$, since $S$ is infinite, $V(a) \cap V(b) \neq \varnothing$. Hence $(a, b) \in V^{2}$, and so $V^{2}=S \times S$. It follows that if for each $V \in \mathscr{Q}, V \subset U, S \times S-V$ is a finite set, then $\mathcal{Q}=\{\delta \times S\}$ and so in either case $\mathcal{Q}$ has a least element.

THEOREM 1. Let ( $S$, Q $)$ ) be a uniform space. Then the following are equivalent conditions:

(a) $(P, \mathcal{V})$ has a totally bounded neighborhood of 0.

(b) $(P, \mathfrak{V})$ has a compact neighborhood of 0.

(c) $(P, \mathcal{V})$ is locally compact.

(d) QL has a least element.

Proof. (a) $\Rightarrow(\mathrm{b})$ : Proposition 4 implies that $\mathscr{Q}$ has a least element $U$. Then $[0,\langle U\rangle]=\{0\}$ is a compact neighborhood of 0 . (b) $\Rightarrow(\mathrm{c})$ : Let $\alpha \in P$; then if $U$ is the least element of $\mathscr{Q}, U^{\prime}(\alpha) \subset V^{\prime}(\alpha)$ for each $V \in \mathscr{Q}$, and so $U^{\prime}(\alpha)$ is a compact neighborhood of $\alpha$. That (c) $\Rightarrow$ (d) and (d) $\Rightarrow$ (a) follows from Proposition 4.

EXAMPLE 1. It is possible for the uniformity to have a least element which is not the diagonal $\Delta$. Let $S=R$ and let $थ$ be the set of all subsets of $R^{2}$ that contain $\Delta \cup\{(-1,1),(1,-1)\}$. Then the set $\Delta \cup\{(-1,1),(1,-1)\}$ is the least element of ข. 
We now turn from local properties of the scale to global properties.

Proposition 5. Let $(S, \mathcal{Q})$ be a uniform space. Then $(P, \mathcal{T})$ is totally bounded if and only if either $S$ is a finite set or $\mathcal{U}=\{S \times S\}$.

Proof. The sufficiency is clear. Suppose that $(P, \mathbb{T})$ is totally bounded and 2 is an infinite set. If there exists an element of $Q$ whose complement is an infinite set, then by Proposition 3, $\mathcal{Q}$ has a least element, say $W$. Then $S \times S-W$ contains an infinite set of $\left(a_{n}, b_{n}\right)$ 's, where $a_{n} \neq a_{m}$ whenever $n \neq m$. Let $\beta_{n}=$ $\left\langle W \cup\left\{\left(a_{n}, b_{n}\right)\right\}\right\rangle, n \geqslant 1$, and $A=\left\{\beta_{n} \mid n \geqslant 1\right\}$. Since $W^{n}=W$, it is straightforward to show that $W^{\prime}(\alpha) \cap A=\{\alpha\}$ for each $\alpha \in A$. Hence $A$ is not totally bounded. It follows that the complement of each element of $\mathcal{Q}$ is a finite set, and thus $\mathscr{Q}=\{S \times S\}$ whenever $S$ is an infinite set.

Note that in Example $1(P, \mathcal{T})$ has a totally bounded neighborhood of 0 , but $(P, \mathscr{T})$ is not totally bounded.

Lemma 1. Let $(S, \mathcal{Q})$ be a uniform space and $V \in \mathcal{Q}$ such that $S-V(x)=A$ for some $x$ in $S$. Let the cardinality of $A$ be infinite. Then $P_{R}$ contains a closed discrete subset of cardinality $2^{\mathrm{card}(A)}$.

Proof. Let $W \in \mathcal{Q}$ such that $W^{3} \subset V$, where $W$ and $V$ are symmetric. Suppose $A=S-V(x)$ for some $x$ in $S$. For each nonempty subset of ( $S-$ $V(x)) \times\{x\}$, let $\alpha_{C}=\langle W \cup C\rangle$ and let $B=\left\{\alpha_{C} \mid C\right.$ is as indicated above $\}$. Then $\operatorname{card}(B)=2^{\operatorname{card}(A)}$.

Let $\alpha \in P$ and suppose that $\alpha_{C}, \alpha_{D} \in B$ such that $C \neq D$ and let $\alpha_{C}, \alpha_{D} \in$ $W^{\prime}(\alpha)$. Assume $D-C$ is nonempty. Since $W$ is symmetric, $\left(\alpha_{C}, \alpha_{D}\right) \in\left(W^{2}\right)^{\prime}$ which implies that $W^{2} \circ(W \cup C) \supset W \cup D$. Let $(a, x) \in D-C$. Then $(a, z)$ $\in W \cup C$ and $(z, x) \in W^{2}$ for some $z$ in $S$. Now, if $(a, z) \in W$, then $(a, x) \in$ $W^{3} \subset V$ which contradicts the fact that $a \notin V(x)$. Therefore, $(a, z) \in C$, which implies that $z=x$ and $(a, x) \in C$. Again we have reached a contradiction because $(a, x) \in D-C$.

Consequently, $W^{\prime}(\alpha)$ contains at most one element of $B$, which implies that $B$ is discrete. We claim that $\bar{B}=\cup\left\{\left(\alpha_{C}\right)_{R} \mid \alpha_{C} \in B\right\}$, where $\bar{B}$ denotes the closure of $B$ in $P$.

Suppose $\left(\gamma, \alpha_{C}\right) \in R$ for some $\alpha_{C}$ in $B$ and $\gamma \notin A$. Since $\gamma R \alpha_{C},\left(\gamma, \alpha_{C}\right) \in U^{\prime}$ for all $U$ in $\mathscr{Q}$, which implies that $\alpha_{C} \in U^{\prime}(\gamma)$ for all $U$ in $\mathscr{Q}$. Hence $\gamma$ is a point of closure of $B$. Our claim follows from the preceding paragraph. 
Since the mapping $\psi$ is both open and closed, the set $\psi(B)(=\psi(\bar{B}))$ is a closed, discrete subset of $P_{R}$. Finally, $\operatorname{card}(\psi(B))=2^{\operatorname{card}(A)}$ since $\psi$ is one to one.

Lemma 2. Suppose $(S, \mathscr{Q})$ is a uniform space where $\operatorname{card}(S)=\alpha$, and $V$ is a symmetric entourage such that for all $x$ in $S, \operatorname{card}(S-V(x))=\beta<\alpha$ where $\alpha$ and $\beta$ are infinite cardinal numbers. Then $V^{2}=S \times S$.

Proof. Since $V(x) \cup(S-V(x))=S$ for any $x$ in $S$, and card $(S-V(x))<\alpha$, then $\operatorname{card}(V(x))=\alpha$. Let $(x, y) \in S \times S$ such that $x \neq y$. Then $V(x) \cap V(y)$ is nonempty because if it were empty, then $S-V(x) \supset V(y)$ which is contrary to the fact that $\beta<\alpha$. Therefore, there exists a $z$ in $V(x) \cap V(y)$ which implies that $(x, y) \in V^{2}$, since $V$ is symmetric. Consequently, $V^{2}=S \times S$.

REMARK. The conclusion of the above lemma remains true if $\alpha$ is infinite and $S-V(x)$ has finitely many elements for all $x$ in $S$.

THEOREM 2. Let $(S, \mathcal{Q})$ be a uniform space. Then the following conditions are equivalent:

(a) $(P, \mathscr{Y})$ is countably compact

(b) $(P, \mathcal{\Upsilon})$ is totally bounded.

(c) $(P, \mathfrak{T})$ is Lindelöf.

(d) $(P, \mathscr{T})$ is compact.

(e) $(P, \mathscr{T})$ is second countable.

(f) $(P, \mathcal{T})$ is separable.

(g) Either $S$ is finite or $\mathscr{Q}_{\mathfrak{L}}=\{S \times S\}$.

Proof. If $S$ is finite or if $\mathscr{Q}=\{S \times S\}$, all the remaining conditions easily follow.

Suppose that $(P, \mathscr{V})$ is countably compact, totally bounded, Lindelöf, compact, second countable, or separable. Suppose that $S$ is not finite. We wish to show that थे $=\{S \times S\}$.

Assume that $\mathscr{Q} \neq\{S \times S\}$. Then by Lemma 2 and the following remark, there exist $V$ in $\mathcal{Q}$ and $x$ in $S$ such that $S-V(x)$ is infinite. By Lemma $1, P_{R}$ contains a closed, discrete subset of cardinality $2^{\operatorname{card}(S-V(x))}$. This fact is contrary to $(P, \breve{Y})$ being countably compact, totally bounded, Lindelöf, compact, or second countable.

Moreover, we claim that it is contrary to $(P, T)$ being separable. Suppose that there is a countable dense subset $E$ of $P$. Choose a symmetric $W \in \mathcal{Q}$ such that $W^{3} \subset V$. Let $B$ be the uncountable subset of $P$ which is defined in the proof of 
Lemma 1. By that proof $W^{\prime}(\alpha)$ contains at most one element of $B$ for each $\alpha \in P$. Since $E$ is a countable dense subset of $P$ and $B$ is an uncountable subset of $P$, there must exist distinct elements $\alpha, \beta \in B$ such that $W^{\prime}(\alpha) \cap W^{\prime}(\beta) \cap E \neq \varnothing$. This implies that $\beta \in W^{\prime}(\alpha)$, a contradiction.

We conclude that $Q=\{S \times S\}$, and the theorem is proved.

Since the retracted scale $P_{R}$ is made up of equivalence classes of elements of the scale $P$, the cardinality of $P_{R}$ must be less than or equal to the cardinality of $P$. For certain uniform spaces the cardinalities are equal.

Let $T$ be a set. In the proof of the following theorem $\mathscr{P}(T)$ will denote the power set of $T$.

THEOREM 3. Let $S$ be an infinite set and $थ$ a uniformity on $S \times S$ such that จ $1 \neq\{S \times S\}$. If $\operatorname{card}(S)=\alpha$, then $\operatorname{card}\left(P_{R}\right)=2^{2^{\alpha}}$.

Proof. Since $P \subset \mathscr{P}(\mathcal{P}(S \times S))$, card $(P) \leqslant 2^{2^{a}}$. By Lemma 2 there exists $V$ in 9. such that $\operatorname{card}(S-V(x))=\alpha$ for some $x$ in $S$. Let $W \in Q$ d such that $W^{3} \subset V$ and let $B=(S-V(x)) \times\{x\}$ where $x \in S$, and $W$ and $V$ are symmetric. Let $F(B)$ denote the collection of all filters on $S \times S$ which contain $B$. Then $\operatorname{card}(F(B))=2^{2^{a}}$. For each $\mathscr{F} \in F(B)$, let $\alpha_{\tilde{\mathscr{F}}}=\langle W\rangle \cap \mathscr{F}$, and $A=\left\{\alpha_{\widetilde{G}} \mid \mathscr{F} \in\right.$ $F(B)\}$. Notice that if $\mathscr{F}, \mathcal{G} \in F(B)$ such that $\mathscr{F} \neq \mathcal{G}$, then $\alpha_{\mathscr{F}} \neq \alpha_{\hat{\mathfrak{G}}}$, which implies that $\operatorname{card}(A)=2^{2^{\alpha}}$. Also, note that $A \subset P$.

We claim that $A$ is discrete. If $\alpha \in P$, and $\alpha_{\bar{q}}, \alpha_{\mathcal{G}} \in W^{\prime}(\alpha)$, then $\left(\alpha_{\bar{q}}, \alpha_{\mathscr{G}}\right) \in$ $\left(W^{2}\right)^{\prime}$ since $W$ is symmetric. If $\mathscr{F}-\mathcal{G}$, we may assume without loss of generality that there exists $F$ in $\mathscr{F}-\mathcal{G}$. For all $G \in \mathcal{G}$ the set $(S-F) \cap G$ is nonempty. Since $\left(\alpha_{\bar{*}}, \alpha_{\hat{G}}\right) \in\left(W^{2}\right)^{\prime}$ and $W \cup F \in \alpha_{\tilde{q}}, W^{2} \circ(W \cup F) \in \alpha_{\mathfrak{g}}$. Hence $W^{2} \circ(W$ $\cup F) \supset W \cup G$ for some $G$ in $\mathcal{G}$. Let $(s, x)$ be a point in $G-F$. Then $(s, z) \in W$ $\cup F$, and $(z, x) \in W^{2}$ for some $z$ in $S$. Now, $(s, x) \notin W^{3} \subset V$ because $(s, x) \in G$ and $G$ is contained in the complement of $V$. Therefore, $(s, z) \in F \subset B$, which implies that $z=x$ and $(s, x) \in F$. We have reached a contradiction to our choice of $(s, x)$; hence, $W^{\prime}(\alpha)$ contains at most one element of $A$. Therefore, $A$ is a discrete subset of $P$, the closure of $A$ in $P$ is $\cup\left\{\left(\alpha_{\tilde{s}}\right)_{R} \mid \tau, F F(B)\right\}$, and the image of the closure of $A$ under $\psi$ is $\psi(A)$.

Now $P$ and $P_{R}$ both contain discrete subsets of cardinality $2^{2^{\alpha}}$, so card $(P)$ and $\operatorname{card}\left(P_{R}\right)$ are at least $2^{2^{\alpha}}$. Recalling that $\operatorname{card}(P) \leqslant 2^{2^{\alpha}}$, we conclude that card $(P)$ $=2^{2^{\alpha}}=\operatorname{card}\left(P_{R}\right)$.

Corollary. Let $(S$, Q $)$ be a uniform space. Then $\operatorname{card}(P)$ and $\operatorname{card}\left(P_{R}\right)$ are either finite or $2^{2^{a}}$. 


\section{References}

[1] N. Bourbaki, Topologie générale, Chapt. I, II (Paris, 1951).

[2] D. Bushaw, 'A stability criterion for general systems,' Math. Systems Theon 1 (1967), 79-88.

[3] D. C. Kent, 'On the scale of a uniform space,' Invent. Math. 4 (1967), 159-164.

[4] G. S. Leslie and D. C. Kent, 'Connectedness in the scale of a uniform space,' $J$. Austral. Math Soc. 13 (1972), 305-312.

[5] G. D. Richardson, 'Connectedness of uniform subspaces of R', J. Austral. Math. Soc. 18 (1974), 46I-463.

Department of Mathematics

East Carolina University

Greenville, North Carolina 27834

U.S.A.
Department of Mathematics

Marshall University

Huntington, West Virginia 25701

U.S.A. 